\title{
Formación de profesores e instructores en materia de nuevas tecnologías de la información.
}

\author{
Vicenta Bustillo Porras \\ supernatavi@mixmail.com \\ Universidad Complutense de Madrid.
}

\section{INTRODUCCIÓN}

En la actualidad el profesor se encuentra en una situación educativa en la que se está produciendo un cambio sustancial en la práctica docente. Los intentos de globalización, interdisciplinariedad, etc., pretenden alcanzar un aprendizaje integrado e integral. Las Nuevas Tecnologías se presentan como recursos facilitadores para producir una enseñanza más dinámica, instrumental y socializadora, acorde con el aprendizaje que se quiere conseguir.

El profesor se constituye en responsable de que la introducción de las nuevas tecnologías en el aula se haga de manera eficiente y eficaz. La utilización de las nuevas tecnologías en los espacios escolares, bien sea desde la concepción de mero recurso, bien sea desde su conversión en puro medio de enseñanza, no consiste en un castillejo de instrumentos tecnológicos, sino en un verdadero armazón que se percibe y funciona con unitariedad produciendo la transformación en la concepción y diseño de los actos didácticos. Para que esto sea posible el profesor está obligado a realizar un análisis serio, técnico y pedagógico a la par que didáctico, sobre los distintos medios. Todos los medios que utilice deben estar organizados y coordinados de forma progresiva y sistemática, configurando lógicas y didácticas orientadas hacia la consecución de los objetivos curriculares.

No debemos olvidarnos que vivimos en una sociedad consumista, donde los clientes más fáciles e indefensos son los más jóvenes. Éste es un problema quizá más peligroso que la propia televisión, ya que ésta se programa al servicio exclusivo del consumo, ofreciéndonos un mundo deseable, cargado de sexo, violencia etc. Las consecuencias son realmente sangrantes: frustración, insatisfacción, agresividad, irreflexión etc.

La publicidad utiliza lo mejor y lo peor de la humanidad con tal de vender lo que sea, convirtiéndose en una auténtica fábrica de necesidades que, a través de la televisión impacta directa y eficazmente en las personas, sobre todo en los que poseen una personalidad más débil. Para que esto no ocurra, hay una gran labor que realizar por parte de los padres, profesores y centros educativos y sociedad, en general, ofreciendo alternativas atractivas y formativas que reduzcan el tiempo en que los niños permanezcan pasivamente frente al televisor.

\section{FORMACIÓN DEL PROFESORADO: INICIACIÓN EN LA PROFESIÓN DOCENTE}

Podemos pensar que cuando hablamos de formación del profesorado nos estamos refiriendo a la iniciación en la profesión docente, o incluso a un preentrenamiento con alumnos que piensan dedicarse a la docencia, pues no es así hay que concebirla en un sentido más amplio, incluyendo el perfeccionamiento. La formación del profesorado la podemos definir, siguiendo a Marcelo, como un proceso sistemático individual o colectivo orientado a la adquisición de conocimientos, destrezas y disposiciones de forma crítica y reflexiva [1] .

La formación del profesorado es un tema controvertido y sobre el que se plantean continuos 
cambios e innovaciones que intentan superar las deficiencias y problemas que se van observando en la formación de los docentes. Estos problemas radican entre otras muchas causas, en el distanciamiento que se produce entre lo que se enseña en las aulas y lo que se necesita a la hora de ejercer como profesor (teoría-práctica) La formación del profesorado consiste en la adquisición de un conocimiento académico mientras que los profesores utilizan a diario un conocimiento de tipo práctico, basado en experiencias profesionales.

Con la incorporación de las Nuevas Tecnologías y las posibilidades que nos ofrecen las Ciencias de la Comunicación e Información, como medio de superar la barrera entre la teoría y la práctica, se puede pensar en las posibilidades que ofrecen las nuevas tecnologías para acercar la formación del profesorado a la realidad que deben afrontar los docentes y en qué medida pueden facilitar la reflexión, auto-observación y auto-análisis, una formación personalizada, animación dinámica de grupos, etc. En definitiva, llevar a cabo actividades que incidan en el perfeccionamiento de la función docente, y por tanto, en el aumento de la calidad de la enseñanza.

Según Beyer, cuanto más tiempo pasa un estudiante en una clase observando y ayudando al profesor mejor es su preparación [2] . Se concibe que este oficio presupone al aprendizaje de destrezas que los alumnos deben lograr, mediante ensayos y errores, durante su formación.

En el comportamental, basado en competencias, la idea principal es descomponer la enseñanza en acciones o comportamientos observables que son objeto de entrenamiento. Permiten un tratamiento individualizado, con materiales autoinstruccionales y pueden ser utilizados equipos multimedia.

En el personalista, basado en el desarrollo personal, se valora en sí mismo como origen de toda conducta, defendiendo que lo que un individuo cree de sí mismo va a afectarle en todos los aspectos de su vida. El profesor debe conocerse bien y reflexionar sobre cómo puede afectar su personalidad a la enseñanza, qué repercusiones puede tener en su relación con los alumnos, modo de solucionar conflictos. Su objetivo principal es el autodescubrimiento personal y tomar conciencia de sí mimo, lograr un autoconcepto positivo, clasificar su mundo de perspectivas y descubrir un modo personal de enseñar.

En el de indagación, se define la importancia de la reflexión e investigación en el aula para conseguir una verdadera innovación en la práctica educativa. Se concibe al profesor como un profesional reflexivo y con un sujeto que tiene que resolver dudas, conflictos que van surgiendo, etc. El profesor tiene que ejercer habilidades para la definición de problemas, proponer soluciones, diseñar procedimientos y obtener evidencias para solucionar las posibles hipótesis, para potenciar la capacidad reflexiva y crítica y poder integrar teoría y práctica.

\section{OBJETIVOS ESPECÍFICOS REFERENTES A LA FORMACIÓN DEL PROFESORADO}

Se pueden establecer criterios básicos para conseguir los objetivos como son el estudio (para la adquisición de conocimientos), el entrenamiento (individual o en grupo para adquirir habilidades) y la reflexión (autoanálisis o discusiones grupales para la adquisición de actitudes)

Todas las estrategias tienen que ser coherentes con los objetivos específicos del mismo, y con las características de los sujetos a quienes va dirigido. Así, podemos citar:

1. Los laboratorios, se puede aprender a través de juegos y simulaciones de clase, donde se toman decisiones interactivas. Se puede observar y decidir sobre diversas situaciones. El material requerido supone la elaboración de casos que permitan apreciar distintos modelos pedagógicos.

2. Microenseñanza, se lleva a cabo en una situación controlada, en la que el profesor desarrolla una situación instructiva en un ambiente menos complejo que el de la clase real; se permite la repetición de las actuaciones hasta conseguir las destrezas. Se ha basado en la modificación de conducta. 
3. Minicursos, mucho más compleja y elaborada que la anterior. Hay que estudiar las habilidades, observarlas, practicarlas y evaluarlas.

4. Talleres, su objetivo es aprender a hacer algo, con una metodología activa: aprender haciendo.

5. Supervisión clínica, hay que modificar la conducta inadecuada del profesor y ayudarle a ser más analítico con su propia práctica, para ello hay que planificar, observar, analizar y comunicar los resultados.

6. Supervisión de compañeros, apoyo técnico y personal a los profesores en el trabajo a través de las críticas constructivas de sus compañeros.

7. Seminarios, reunión de un grupo de profesores para analizar las dificultades que encuentran en su práctica educativa y proporcionar un cambio de actitudes.

8. Simulación, actuación en un sistema ficticio y simplificado que permite la toma de decisiones en una situación de seguridad para el sujeto y una supervivencia directa. Se puede conseguir mediante role-playing, estudio de casos, materiales de protocolo, etc. Su objetivo es entregarse a la toma de decisiones y a la reflexión de la realidad.

9. Etnografía e investigación-acción, se centran en el análisis crítico de la realidad, la exploración de los conflictos entre valores y conductas, para diagnosticar y solucionar un problema en un contexto determinado; para esto se lleva a cabo observación, entrevista y recogida de datos.

Definir qué debe o puede ser, cómo puede o debe articularse y qué implicaciones organizativas y curriculares pueden tener las Tecnologías de la Información y de la Comunicación en la formación inicial del profesorado, forman parte del marco cultural de nuestra sociedad y han penetrado en todos los campos de la actividad humana: desde el productivo al del ocio programado; estas tecnologías se manifiestan, con mayor o menor intensidad, produciendo cambios de perspectiva sobre la naturaleza de las cosas y ofreciendo la posibilidad de reconstrucción de la realidad a partir de ciertos modelos que, con más o menos pretensiones, buscan representar con fidelidad.

En este sentido, la enseñanza escolar no ha quedado al margen de estos cambios, algunos de los cuales se han producido con tal aceleración que han superado la capacidad de compresión colectiva de las transformaciones producidas.

Para Pérez [3] , el profesorado ha de conocer el marco curricular, ejemplos y alternativas de proyectos curriculares, la estructura epistemológica del ámbito y el grupo social de estudiantes, y a partir de estos elementos presenta los siguientes aspectos para una formación del profesorado [4] :

Adquisición de un bagaje cultural de clara orientación política y social

Desarrollo de capacidades de reflexión crítica sobre la práctica

Desarrollo de actitudes que requieren compromiso político del profesorado como intelectual transformador en el aula, en la escuela y en el contexto social.

Blázquez, Cabero y Loscertales [5], nos hablan de los tres pilares fundamentales de la formación del profesorado:

La formación científico-cultural

La formación psicopedagógica y didáctica

La reflexión sobre la práctica de la formación 
La finalidad de la formación del docente no es sólo que éste aprenda, sino que aprenda a enseñar [6] , y el objetivo fundamental de la enseñanza es enseñar a aprender, el alumno ha de aprender a aprender, y que este aprendizaje se realice basándose en una formación científico-cultural, psicopedagógica y didáctica, y a una reflexión sobre la práctica de la formación.

Los modelos de formación del profesorado deben de ir desde la perspectiva académica, pasando por la técnica y de ahí a la práctica. La historia de la formación del profesorado en nuevas tecnologías ha tenido desde sus inicios un marcado sesgo tecnológico y de un olvido de la metodología y la reflexión sobre los aspectos psicopedagógicos. Esto poco a poco ha ido mejorando, aunque los avances son aún tímidos.

\section{EJES EN LA FORMACIÓN DEL PROFESORADO EN NNTT}

Como propuesta concreta, una concreción racional de los ejes de la formación en nuevas tecnologías puede ser [7] :

\begin{tabular}{|c|c|c|}
\hline Eje & Contenido & Ámbito \\
\hline 1 & Formación científica y cultural & Conocimientos previos \\
\hline 2 & $\begin{array}{c}\text { Formación didáctica, metodológica y de } \\
\text { recursos de las nuevas tecnologías }\end{array}$ & Propio de la asignatura \\
\hline 3 & $\begin{array}{c}\text { Implicaciones de las nuevas tecnologías en los } \\
\text { procesos de enseñanza y aprendizaje }\end{array}$ & Propio de cada materia \\
\hline 4 & $\begin{array}{c}\text { Conocimiento de los currículos y de los ejes } \\
\text { transversales }\end{array}$ & Propio de cada materia \\
\hline 5 & $\begin{array}{c}\text { Creación de actividades de aprendizaje } \\
\text { Complementado en la asignatura }\end{array}$ \\
\hline 6 & $\begin{array}{c}\text { Reflexión sobre los usos de los medios y su } \\
\text { relación con modelos curriculares y enfoques } \\
\text { metodológicos }\end{array}$ & Propio de la asignatura \\
\hline 7 & Reflexión sobre la práctica & De todas las asignaturas \\
\hline
\end{tabular}

La enseñanza, en tanto que subsistema del sistema social, debe incorporar los logros técnicos que esa misma sociedad a la que pertenece desarrolla e incorpora en otros contextos. Los profesores deben disponer de la formación y de los medios necesarios, desde la perspectiva comunicativa de éstos, como desde la didáctica y la funcional. Las funciones del docente prestan atención a aquellos que tienen que ver con los procesos de comunicación y con la información. La acción didáctica del profesorado precisa la incorporación de medios y recursos que colaboran en el logro de los objetivos previstos.

El mundo de la enseñanza ha incorporado buena parte de esas posibilidades comunicativas a sus diseños curriculares, unas veces por necesidad, y otras por presiones extraescolares. Por suerte, hoy en día, la distancia y las condiciones geográficas no suponen ninguna limitación, debido a los canales de comunicación. Esta posibilidad de eliminar las distancias, tanto para la acción como para el traslado de unos contenidos concretos, está transformando los elementos básicos de la comunicación humana, configurando nuevos espacios comunicativos y laborales. La teleconferencia y el teletrabajo, son algunas de estas posibilidades que están teniendo repercusiones en el ámbito de la enseñanza, transformando la idea de aula escolar tal y como la entendemos en este momento.

La enseñanza de las nuevas tecnologías en tanto que conocimiento en sí, tiene dos niveles bien diferenciados, uno de especialización para los docentes que se ocupan de la enseñanza de estas temáticas y uno general para aquellos que van a ser usuarios. Importante tener en cuenta las capacidades necesarias para poder ser usuarios de las nuevas tecnologías en cualquier ámbito del 
trabajo. No hay que pasar por alto los aspectos pedagógicos de la acción docente y, dentro de ellos, los didácticos. Buena parte de las Nuevas Tecnologías tienen que ver con la comunicación y la información y es evidente que el profesor ha de tener la formación necesaria sobre las posibilidades que las nuevas tecnologías ofrecen, de forma que pueden tomar decisiones sobre su incorporación a los diseños de acciones concretas que planifique. Se trata de contemplar las tecnologías como medios de enseñanza, entendidos de forma general y que por ello deberá ser un objetivo a lograr con la totalidad del profesorado [8] .

Hay que hablar de dos tipos de formación: la que es fruto del ejercicio de la profesión y de la reflexión sobre el mismo, tanto personal como grupal, y la que se establece como algo añadido, pero convergente con la primera.

El MEC y D [9] recomienda que para garantizar un mayor ajuste y atención a las necesidades reales del profesorado así como su implicación en los procesos de formación, se debe incrementar la participación de los representantes de los centros docentes en las actividades que relacionadas con la planificación, corresponden a los consejos de los centros de profesores, hay que mejora su quehacer docente, lo que obliga a insistir muy poco en los aspectos más tecnológicos y profundizar en lo referente al uso y posibilidades comunicativas de las nuevas tecnologías, así como en las implicaciones didácticas y en los diseños que se incorporan.

La formación debería darse de una manera general para el profesorado que no haya tenido esa oportunidad en su formación inicial, y luego para todos ellos, ofrecer la posibilidad cada vez que existan avances relevantes o significativos, de que puedan adquirir los conocimientos necesarios. Posteriormente se requerirá una formación acorde con el desarrollo que se vaya produciendo en estas tecnologías, con los docentes que desempeñan funciones dentro de la teleenseñanza, profesionales que si en estos momentos apenas existen, será necesario pensar en su formación. No olvidarnos de los alumnos de los que nos hemos de ocupar, ya que en el campo de la educación especial, las funciones de estas tecnologías poco tienen que ver con las que corresponden a aulas convencionales.

\section{EL PROFESOR COMO FUENTE DE INFORMACIÓN E INFORMADOR RESPECTO A SU FORMACIÓN EN NNTT}

La introducción de los medios de comunicación en el aula suponen un cambio importante en las relaciones profesor alumno, rompiendo una tradición pedagógica según la cual el maestro es la única fuente de información de la que debe nutrirse el alumno. Aunque el libro de texto ya rompió parcialmente tal monopolio, su menor poder de atracción raramente lo convirtió en fuente substitutiva de la información docente. Seguramente en la superación del monopolio informativo del profesor puede residir la clave del problema que nos ocupa.

El profesor no debe competir con otras fuentes informativas, sino erigirse en elemento aglutinador y analizador de todas esas fuentes incluyéndose él mismo como informador.

El profesor inevitablemente, ha de convertirse en planificador educativo, que dispone de una amplia variedad de recursos para satisfacer las necesidades de sus alumnos. Las innovaciones técnicas han de ser usadas dentro del proceso de enseñanza-aprendizaje con flexibilidad, pero sin regateos, para lograr síntesis de conocimientos y, sobre todo, hábitos cognitivos.

La habilidad de informarse a través de múltiples fuentes aparece como objetivo prioritario. Luego se añade el conocimiento y, en la media de lo posible, dominio de los diversos medios de comunicación. La enseñanza en materia de comunicación es algo que pueden realizar eficazmente los profesores, lógicamente, tras recibir una preparación adecuada; tal preparación cabría situarla en la formación inicial de los futuros profesores y en la permanente para los que ya estén en ejercicio. Ambos objetivos son urgentes, si se desea arreglar el abismo que hay entre los intereses y prioridades de la mayoría de los centros por un lado, y los problemas, situaciones y asuntos concretos que, por el otro, viven alumnos y adultos en el mundo de hoy. 
La escuela ya no deberá contentarse con difundir directamente los conocimientos, sino que deberá sobre todo, ocuparse de estructurar los adquiridos por otros medios, por los alumnos y enseñarles a reunir y seleccionar por sí mismos la información o más bien prepararlos para ello. Los alumnos no tendrán que aprender menos que antes, sino que deberán aprender otras cosas, sirviéndose de medios diferentes. La información se impone en un proceso irreversible, conformando el modo de conocer el estilo cognitivo y ofreciendo un nuevo estilo, una forma de pensar y de trabajar [10].

\section{MODIFICACIÓN DEL PAPEL DEL PROFESOR ANTE LAS CIRCUNSTANCIAS INTERNAS Y EXTERNAS DEL SISTEMA EDUCATIVO}

Hay que formar a los profesores para que sean capaces de explotar las posibilidades educativas de la programación general de los medios, leer sus lenguajes y captar los supuestos axiológicos implícitos en sus mensajes. Significa, a la vez, formar a los comunicadores, para hacerles sensibles a la satisfacción de las demandas que parten del sistema educativo y de la sociedad en general respecto a contenidos y programas enriquecedores en conocimientos y valores.

El papel de profesor se ve modificado frecuentemente por las circunstancias externas globales o locales así como por las internas del sistema educativo e incluso del respectivo centro de enseñanza, con repercusión tanto en metas como en contenidos, métodos o medios de enseñanza e investigación. Esta dinámica de cambio es particularmente visible hoy en día y la presión exterior sigue en aumento. Sin embargo, es muy poco necesario cambiar el fundamental papel orientador, educador, enseñante y tutor del buen docente de siempre.

La reflexión sobre la formación y actualización del profesorado de educación secundaria y sobre su papel de futuro debería ir dirigida de modo prioritario a los docentes de la educación formal, es decir, los profesionales; pero también, de alguna manera, a todos los demás docentes no profesionales en una sociedad civil consciente de su mancomunada responsabilidad educadora [11] .

La lista de instructores es larga y ninguno sobra. Empiezo por mencionar el decisivo papel educativo que para todo creyente tienen sus referencias e instituciones religiosas. La aportación formadora de los instructores para la defensa nacional también es reconocida por las sociedades que viven en democracia. A ello se suma el reconocimiento a la creciente labor educativa en el seno de las empresas privadas dedicadas a la producción o al comercio, como lo atestiguan ejemplos significativos y abundantes en los países más desarrollados. No quedan excluidos de responsabilidad educativa los políticos a la hora de formular mensajes y de proponer programas para la acción. Ni puede olvidarse, sobre todo, la labor educativa que ejercen los artistas todos, empezando por los escritores, así como las instituciones culturales más diversas. Sin embargo, destaca entre todos estos actores, directos o indirectos, de la educación informal y no formal, el creciente papel de los verdaderos, de los buenos periodistas cuando informan coherente y verazmente, y tanto más cuanto ofrecen opinión desde la perspectiva de sus particulares criterios, los cuales deben exponer siempre de forma explicita y clara, para dejar total libertad a quienes tratan de informarse y formarse en tales lecturas.

La tarea docente necesaria es inmensa y muy difícil, [12] esa dificultad es proporcionalmente mayor, en su misión formativa, en los primeros niveles de la enseñanza, cuando realmente se ponen las bases esenciales para el futuro de cada persona en un proceso extraordinariamente delicado y complejo, si bien la dificultad científica aumenta a medida que se avanza en el nivel de conocimientos que se pretende transmitir.

La introducción de cambios en los centros educativos así como en las instituciones de formación del profesorado es siempre compleja, y relativamente lenta. Integrar las tecnologías de la comunicación y de la información en el proceso de enseñanza y aprendizaje sigue siendo difícil a pesar de años de esfuerzos y de los rápidos progresos alcanzados durante los últimos tiempos.

La competencia científica y pedagógica del docente de hoy y del mañana tiene que ir muy de 
la mano de su profesionalidad y de su dedicación y creatividad ante esa relación vocacional con sus alumnos, la cual supone ganarse su respeto y afecto. A tan fin, y pese a que el tiempo es el recurso más limitado de todos los que disponemos, el profesor tiene que poder dedicar mucho tiempo, que no siempre se le concede y reconoce, como parte de su horario de trabajo, pese a ser algo esencial para el ejercicio de su primordial función tutora, orientadora.

\section{LA DOCENCIA EN EL CONTEXTO DE LA DIVERSIDAD CULTURAL Y LINGÜÍSTICA}

Los modelos de formación del profesorado siguen necesariamente abiertos a una gran diversidad de situaciones y necesidades, empezando por la diversidad del medio cultural y tanto más ante los casos de multiculturalidad. La docencia, en el contexto de la diversidad cultural y lingüística, tiene que saber superar los resabios racistas, xenófobos y del nacionalismo exacerbado, además de transmitir actitudes y hábitos positivos, junto con saberes y habilidades. Para poder cumplir adecuadamente estas funciones es imprescindible mejorar la capacidad del profesorado para enseñar en un contexto multicultural, aprovechando las experiencias disponibles en diversos países, las cuales van desde la investigación a la incorporación de programas para aprender a apreciar la diversidad y superar el etnocentrismo así como las actitudes negativas hacia las minorías.

Atención particular requiere la formación del profesorado para la formación profesional y técnica al servicio del desarrollo [13] , puesto que además de la formación en la especialidad y sin desmedro de una base cultural similar a la de los demás docentes del respectivo nivel educativo, requiere una didáctica específica.

Es la sociedad, y muy concretamente los responsables de la política educativa en todos sus niveles y ámbitos territoriales, los que tienen que permitir, proporcionar y desarrollar la más plena participación de los docentes en múltiples funciones: en la formación de nuevas metas, en el diseño de la organización escolar, en los planes de formación y actualización en servicio del propio profesorado, en el desarrollo de métodos pedagógicos y en la introducción de nuevas tecnologías y materiales educativos, así como en la evaluación de resultados y rendimientos.

Como dice J.C. Tedesco [14] , los docentes son tratados bien sea como víctimas de la sociedad y de los sistemas educativos (pedagogía dominante), como culpables del deficiente rendimiento educativo (pedagogía crítica), o simplemente se subestima su papel frente al futuro predominante de las nuevas tecnologías.

Hay que lograr asociar de una vez por todas a los docentes en cuantas decisiones educativas les atañen y en las que tanto se espera de su eficacia, creando a tal fin los mecanismos más sencillos posibles. [15]

El informe Delors [16] nos dice que "la sociedad tiene que dotar al profesorado de la autoridad necesaria y de los adecuados medios de trabajo". El profesorado tiene que estar siempre en el centro de cualquier cambio en materia educativa.

Las funciones docentes se centran en las labores de orientación, asesoramiento y tutorización, siempre y cuando se cumpla la premisa de que sean los medios didácticos los encargados de transmitir la información y de facilitar las condiciones de aprendizaje de los alumnos y, además, por parte de éstos se tome conciencia de la necesidad del estudio independiente como punto de partida de su aprovechamiento en esta modalidad de enseñanza.

El desarrollo de las funciones docentes no puede abordarse sólo desde la base de una sólida formación inicial. Los profesores tienen que intervenir en la selección de los materiales y contenidos que han de formar parte de sus programas y elaborar o participar en la construcción y/o adecuación de los medios didácticos que se vayan a utilizar. Hay que reconocer que la principal vía que tienen los educadores para aprender a enseñar es su propia vivencia como alumnos, respondiendo al modelo que aplicaron sus profesores. Por eso, es importante promover su participación en cursos de actualización 
profesional, en los que se empleen Nuevas Tecnologías. Pongamos por ejemplo, el curso "on line teaching", que imparten la universidad de Londres y la Open University a través de sistemas de conferencia electrónica, y en el que toman parte profesores y educadores distribuidos en todo el mundo. En el ámbito nacional, merece especial atención la iniciativa del programa de informática educativa de la Generalitat, que viene desarrollando cursos a distancia basados en soportes telemáticos dirigidos al profesorado de enseñanza primaria y secundaria [17] .

Para la formación de los docentes, la experimentación práctica en los propios centros de enseñanza parece la modalidad más prometedora. Las acciones de formación deberán acompañar al desarrollo de la red de escuelas e implicar, a largo plazo, a un gran número de docentes, pero también de instrucciones, habida cuenta de la semejanza de los métodos. La formación abarcará la utilización de las herramientas, la investigación y la estructuración de la información a partir de las redes, y la concepción y difusión de contenidos multimedia pedagógicos. Se reforzará mediante servicios de apoyo técnico y pedagógico que puedan ayudar a los profesores, una vez formados, a la utilización en las aulas de los multimedia educativos. De lo que se trata es de:

- Reforzar las acciones de formación en el marco de los programas comunitarios, los intercambios entre enseñantes de escuelas europeas, para poder compartir experiencias, y fomentar el desarrollo de tele- prácticas al objeto de permitirles adquirir estas experiencias profesionales sin desplazarse, mediante la utilización de herramientas de formación a distancia.

La creación de una red de centros de formación de profesores e instructores basada en las iniciativas locales y nacionales, así como en los proyectos comunitarios existentes.

\section{BIBLIOGRAFÍA}

MARCELO, C. (1989): Introducción a la formación del profesorado. Teoría y métodos. Servicio de Publicaciones de la Universidad de Sevilla. Sevilla.

BEYER, L.E. (1990): Field experience ideology and the devolopment of critical reflexivity. Journal of Teacher Education, 35(3), pp. 36 - 41.

BIE (1996): Conferencia Internacional de Educación, 45 reunión. Declaración. Ginebra.

BLÁZQUEZ, F., CABERO, J. y LOSCERTALES, F. (coords.) (1994): Nuevas Tecnologías de la Información y la Comunicación para la educación. Alfar. Sevilla.

COMISIÓN INTERNACIONAL SOBRE LA EDUCACIÓN PARA EL SIGLO XXI (1992), presidida por Jacques DELORS, O. Jacob. París.

DÍEZ HOCHHLEITNER, R. (1997): El docente ante la escuela del futuro. Jornadas de Formación del Profesorado de Cantabria: Educación y Sociedad, una mirada hacia el futuro. Santander.

DÍEZ HOCHLEITNER, R. (1997): Los educadores ante el futuro. Congreso de Pedagogía: Atreverse a educar. Pedro POVEDA ( educador ). Narcea, Madrid.

DÍEZ HOCHLEINER, R. (1997): Educación y desarrollo. Fundación Santillana, Madrid.

GIMENO, J y PÉREZ, A.I. (1992): Comprender y transformar la enseñanza. Morata. Madrid.

GIMENO, J y PÉREZ, A.I. (1994): La función profesional del docente al final del siglo. Conflictos de perspectiva. Escuela Crítica, n. ${ }^{\circ} 7$, Madrid.

MEC (1994): Plan nacional de formación permanente del profesorado. Curso 1994-95. MEC, Madrid. 
PÉREZ, A.I. (1994): LA FUNCIÓN Y FORMACIÓN DEL PROFESOR/A EN LA ENSEÑANZA PARA LA COMPRENSIÓN: DIFERENTES PERSPECTIVAS. ESCOLA CRÍTICA, N. 7 , MADRID.

PÉREZ, A.I. (1986): Más sobre la formación del profesorado. Cuadernos de Pedagogía, n. ${ }^{\circ}$ 139, Madrid.

PÉREZ GÓMEZ, A. (1987): El pensamiento del profesor. Vínculo entre la teoría y la práctica. Revista de Educación, $\mathrm{n}^{\circ} 284$.

PÉREZ GÓMEZ, A. (1988): Más sobre la formación del profesorado. Cuadernos de Pedagogía, n $^{\circ}$ 139.

PÉREZ GÓMEZ, A. (1988): Autonomía y formación para la diversidad. Cuadernos de Pedagogía, $\mathrm{n}^{\circ}$ 161.

PÉREZ, R. y otros (1988): Actitudes del profesorado hacia la incorporación de las Nuevas Tecnologías de la Comunicación en educación. ICE. Servicio de Publicaciones de la Universidad de Málaga. Málaga.

PÉREZ, A. y CALVO, A. (1994): Sistemas de formación ocupacional a distancia. Pixel-Bit, nº 3.

PÉREZ SERRANO, G. (1984): El análisis de contenido de la prensa. La imagen de la Universidad a Distancia. UNED, Madrid.

SANTOS, M.A. (1996): La formación inicial. El currículum del nadador. Cuadernos de Pedagogía, n. ${ }^{\circ} 220$.

SANCHO, J.M. y MILLÁN, L. M. (1995): Hoy ya es mañana. Tecnologías y educación: un diálogo necesario. M.C.E.P., Sevilla.

TEDESCO, J. C. (1997): Fortalecimiento del rol de los docentes. Congreso de Pedagogía: Atreverse a educar. Pedro POVEDA (educador), Narcea, Madrid.

VIORRETA, C. y MARTÍN DE LA HOZ, P. y otros (1994): Formación permanente del profesorado en modalidad a distancia basada en soportes telemáticos en EMI, International Council for Educacional Media (ICEM), Vol 30, $\mathrm{n}^{\circ} 4$.

\section{Notas:}

[1] MARCELO, C. (1989): Introducción a la formación del profesorado. Teoría y métodos. Servicio de Publicaciones de la Universidad de Sevilla. Sevilla.

[2] BEYER, L.E. (1990): Field experience ideology and the devolopment of critical reflexivity. Journal of Teacher Education, 35(3), pp. 36 - 41.

[3] PÉREZ, A.I. (1986): Más sobre la formación del profesorado. Cuadernos de Pedagogía, n. ${ }^{\circ}$ 139, Madrid, pp. 92 - 94

[4] PÉREZ, A.I. (1992): La función y formación del profesor/a en la enseñanza para la comprensión: diferentes perspectivas. En Gimeno, J. y Pérez, A.I.: Comprender y transformar la enseñanza. Morata. Madrid.

[5] BLÁZQUEZ, F., CABERO, J. y LOSCERTALES, F. (coords.) (1994): Nuevas Tecnologías de la Información y la Comunicación para la educación. Alfar. Sevilla. 
[6] SANTOS, M.A. (1996): "La formación inicial. El currículum del nadador. Cuadernos de Pedagogía, n. ${ }^{\circ} 220$, pp. $50-54$.

[7] SANCHO, J.M. y MILLÁN, L.M. (1995): "Hoy ya es mañana. Tecnologías y educación: un diálogo necesario M.C.E.P., Sevilla, pp. 313.

[8] VILLAR, L.M. (1994): La formación del profesorado en Nuevas Tecnologías. En BLÁZQUEZ, F.; CABERO, J. y LOSCERTALES, F: Opus Cit.

[9] MEC (1994): Plan nacional de formación permanente del profesorado. Curso 1994-95. MEC, Madrid, pp. 11 y ss.

[10] PÉREZ SERRANO, G. (1984): El análisis de contenido de la prensa. La imagen de la Universidad a Distancia. UNED, Madrid, pp. 1 y ss.

[11] DÍEZ HOCHHLEITNER, R. (1997): El docente ante la escuela del futuro. Jornadas de Formación del Profesorado de Cantabria: Educación y Sociedad, una mirada hacia el futuro. Santander.

[12] DÍEZ HOCHLEITNER, R. (1997): Los educadores ante el futuro. Congreso de Pedagogía: Atreverse a educar. Pedro POVEDA ( educador ). Narcea, Madrid.

[13] DÍEZ HOCHLEINER, R. (1997): Educación y desarrollo. Fundación Santillana, Madrid.

[14] TEDESCO, J.C. (1997): Fortalecimiento del rol de los docentes. Congreso de Pedagogía: Atreverse a educar. Pedro POVEDA (educador), Narcea, Madrid.

[15] BIE (1996): Conferencia Internacional de Educación, 45 reunión. Declaración. Ginebra.

[16] Comisión Internacional sobre la Educación para el siglo XXI, presidida por Jacques DELORS: Opus Cit.

[17] VIORRETA, C. y MARTÍN DE LA HOZ, P. y otros (1994): Formación permanente del profesorado en modalidad a distancia basada en soportes telemáticos en EMI, International Council for Educacional Media (ICEM), Vol 30, nº 4. 